\title{
RAPID PROTOTYPING OF A TEST MODEM FOR TERRESTRIAL BROADCASTING OF DIGITAL TELEVISION
}

\author{
Ana García Armada and Miguel Calvo Ramón \\ Grupo de Radiación, Dpto. SSR \\ Polytechnic University of Madrid (SPAIN) \\ e-mail: ana@gr.ssr.ump.es
}

\begin{abstract}
This paper introduces Rapid Prototyping of a complex system and the difficulties that appear when a real-time prototype of such a system is approached. These concepts are applied to the construction of a test modem for Terrestrial Broadcasting of Digital Television, in which Orthogonal Frequency Division Multiplexing (OFDM) is the transmission technique.
\end{abstract}

The possibility of reducing the number of sub-carriers in the OFDM signal and obtaining enough information about the system performance in hostile environments (multipath, phase noise, non-linear, ...) is considered and discarded. As a conclusion, a non-real time prototype is presented which allows one to optimize parameters, verify the possibility of implementation and evaluate subjective qualities.

\section{INTRODUCTION}

The research in the area of VHF/UHF terrestrial broadcasting is coming nowadays to a completely digital implementation both in the US (Grand Alliance) [1] and Europe. The future digital systems must be compliant with very strict requirements in terms of bit error rates. Besides, they must fight multipath, be friendly to existing analog systems, cope with the interference caused by them [2] and fit in the assigned available bandwidths.

The complexity involved in achieving these objectives forces us to test and optimize the system before its final implementation. This is normally done by means of analysis and simulation, postponing the construction of prototypes to the last steps in the design cycle. However, prototyping in earlier stages allows us to:

- test more parameters in a shorter time

optimize parameters in real time conditions

- evaluate subjective characteristics

- verify the possibility of implementation.

For these reasons there is an increasing interest in Rapid Prototyping of communications systems, with the main objective of reducing the design cycle and improving the products quality.

In the European DVB Project (Digital Video Broadcasting), founded by initiative of more than 190 organizations, the standards for digital television broadcasting have been specified for the different transmission media: satellite (DVB-S), cable (DVB-C) and terrestrial (DVB-T) $[3,4]$.

The later is a result of the RACE Project dTTb (Digital Television for Terrestrial Broadcasting), which has covered all the aspects of digital television terrestrial broadcasting in Europe, with the work of 30 partners from 10 different countries [5].

The system specified within the dTTb Project is very complex [4,5] (see Figure 1 and Figure 2). Taking as an input the signal coming from an MPEG-2 encoder [6], all the elements for its correct transmission and reception are defined, adapting the signal to the terrestrial channel. With this aim, a combined encoding and modulation process [7] is applied to the data source, with different coding rates $[8,9]$ (which imply different bit rates), according to the channel characteristics and the expected quality at the receiver. Besides, different services, depending on the quality of the received TV signal (standard, enhanced and high definition), several configurations (by combination of these services) and various coding schemes are defined.



Figure 1 dTTb transmitter block diagram

To summarize, transmitter and receiver must be very flexible and this can only be achieved if most of the elements are implemented in software. This idea leads to the Software Radio concept $[10,11]$ in which as many functions as possible are programmed by bringing $A / D$ and $\mathrm{D} / \mathrm{A}$ converters [12] the closer to the antenna.

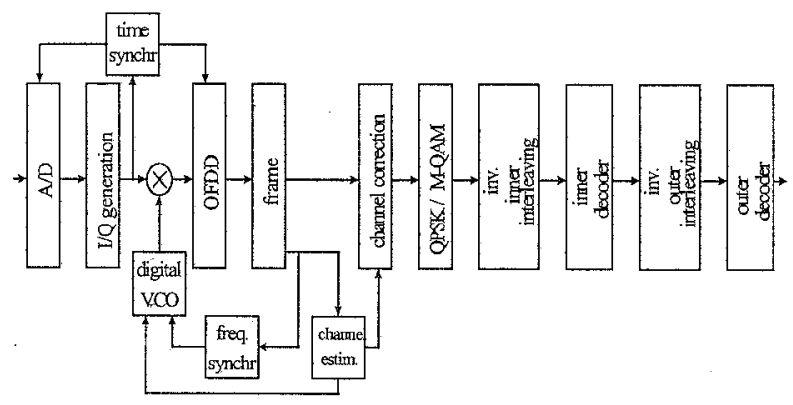

Figure 2 dTTb receiver block diagram

In this context, we have developed a methodology to allow the use of software design tools to implement in a rapid and efficient way communications systems prototypes using digital signal processors (DSPs). This 
methodology has been applied to the construction of a prototype for the European Digital Television for Terrestrial Broadcasting System (dTTb).

None of the published works in this field [13-18], has approached the application of these concepts to such a system. However, its complexity and the fact that we are dealing with rapidly emerging technologies configure it as a clear example of the Rapid Prototyping advantages.

Rapid Prototyping of a complex system presents some difficulties, which depend on the system characteristics. In the next section, the transmission technique used in the DVB-T standard (Orthogonal Frequency Division Multiplexing) is introduced and the problems associated with the construction of a prototype are identified. We propose two different solutions and discuss their feasibility.

Finally, the solution adopted and the prototype constructed using two Ariel MM-96 (board which includes two Motorola DSP96002) are presented.

\section{RAPID PROTOTYPING OF AN OFDM-BASED SYSTEM}

The European system for Terrestrial Broadcasting of Digital Television has strong requirements. It has to be able to cope with multipath, friendly and robust to existing analog systems and make efficient use of the available bandwidth. This reasons have led to the adoption of the Orthogonal Frequency Division Multiplexing (OFDM) technique for the signal transmission.

In this section, the OFDM concept is introduced and the problems associated to its rapid implementation are identified.

\subsection{Orthogonal Frequency Division Multiplexing}

Among the activities of the latest European RACE and ACTS projects, there has been an increasing interest in OFDM (Orthogonal Frequency Division Multiplexing) as a means of combating impulsive noise and multipath effects and making fuller use of the system available bandwidth.

OFDM is one of the best alternatives to alleviate the multipath effects in mobile communications [19]. For reception in built-up areas with delay spreads of some microseconds, the channel is highly frequency selective and sophisticated equalization techniques are required to achieve high bit rate transmissions. However, a combination of OFDM and coding associated with interleaving in the frequency domain (COFDM) can take advantage from the diversity associated to multipath [20]. Besides, multi-carrier systems have been proved to be more computationally efficient than singlecarrier systems with equalization under some multipath channel conditions [21].

In an OFDM system the spectrum associated to each elemental data is a small portion of the total bandwidth, which is divided in $N$ sub-channels. Each of them is modulated with one symbol and they are all multiplexed in frequency.

If $T$ represents the OFDM symbol duration, that is: $T=\frac{1}{B \cdot N}$, the $N$ carriers are placed in the frequencies:

$$
f k=f_{o}+k / T, \quad k=1,2, \ldots, N-1
$$

A higher spectral efficiency is achieved because the different sub-channels are allowed to overlap. Orthogonality between them simplifies the separation process in the receiver, allowing the use of the Discrete Fourier Transform [22].

\subsection{Implementation of OFDM using the Discrete Fourier Transform}

The complex envelope of the transmitted OFDM signal, sampled with sampling frequency $f_{s}=\frac{1}{B}$, is:

$$
s(n)=\sum_{m=-\infty}^{\infty} \sum_{n=N_{g}}^{N-1} \sum_{k=0}^{N-1} s_{m, k} \cdot e^{j \frac{2 \pi}{N} k n} \prod\left(n-m\left(N+N_{g}\right)\right)
$$

This means that every OFDM symbol has $N+N g$ values which are the $N$ obtained by an $N$-point Inverse Discrete Fourier Transform (IDFT) plus an added Time Guard of $N_{g}$ (the last $N_{g}$ samples of the IDFT are added at the beginning of each symbol) in order to cope with the inter-symbol interference (ISI) caused by multipath delay spread. $\Pi(\mathrm{n})$ is a rectangular pulse of duration $N+N g$ samples.

The Time Guard is eliminated in the receiver and in the absence of channel or noise impairments, the signal is Orthogonal Frequency Division Demultiplexed in the receiver by means of an $\mathrm{N}$-point Discrete Fourier Transform (DFT):

$$
\begin{gathered}
\frac{1}{N} \sum_{r=0}^{N-1} s(r) \cdot e^{-j \frac{2 \pi}{N} r m}=\frac{1}{N} \sum_{r=0}^{N-1} \sum_{k=0}^{N-1} s_{k} \cdot e^{j \frac{2 \pi}{N} k r} \cdot e^{-j \frac{2 \pi}{N} r m}= \\
=\frac{1}{N} \sum_{k=0}^{N-1} s_{k} \sum_{r=0}^{N-1} e^{j \frac{2 \pi}{N} r(k-m)}=s_{k}
\end{gathered}
$$

because

$$
\frac{1}{N} \sum_{r=0}^{N-1} e^{j \frac{2 \pi}{N} r(k-m)}= \begin{cases}1 & k-m=c N, c \text { integer } \\ 0 & \text { otherwise }\end{cases}
$$

Summarizing, the OFDM and OFDD processes involve an inverse DFT in the transmitter and a DFT in the receiver. Both can be efficiently implemented by using the Fast Fourier Transform algorithm (FFT).

\subsection{Rapid Prototyping of OFDM}

OFDM has been proposed and is being tested for the Digital Television for Terrestrial Broadcasting System (dTTb) [5] considering two possible values for the number $N$ of sub-carriers: $2048(2 \mathrm{~K})$ and $8192(8 \mathrm{~K})$. The sampling frequency in this system is $f_{s}=64 / 7 \mathrm{MHz}$ $=9.142857 \mathrm{MHz}$, which means that the sub-carrier spacing $\Delta f=f_{s} / N$ and the symbol time $T$ are: 
- 8K mode: $\Delta f=1.116 \mathrm{KHz}, T=896 \mu \mathrm{s}$.

- 2K mode: $\Delta f=4.464 \mathrm{KHz}, T=224 \mu \mathrm{s}$.

Real Time Prototyping of a system with these parameters implies implementation of $2 \mathrm{~K}$ and $8 \mathrm{~K}$ FFTs in the OFDM symbol time. However, the MM-96 with a $40 \mathrm{MHz}$ clock has a benchmark of $2420 \mu$ s for a Radix 2 Cooley-Tukey Decimation in Time complex FFT of $2 \mathrm{~K}$ points and $11320 \mu$ s for a $8 \mathrm{~K}$ one $[23,24]$.

Besides, Rapid Prototyping implies that the code will not be thoroughly optimized, and the use of several processors in parallel will exhibit inefficiencies which will not allow us to obtain a maximum speed-up [25].

These circumstances lead to the consideration of the impossibility of real time implementation with a low number of DSPs. We propose two alternative solutions:

(1) Scaling some parameter in the system so that a real time implementation is possible:

Depending on the system characteristics, the reduction of some parameter can lead to a realizable prototype without variation of the main system properties. In our case, we will try reducing the number of sub-carriers and study the implications in the OFDM signal characteristics.

As Figure 3 shows, a system with 128 sub-carriers could be a good approach to a real time implementation.

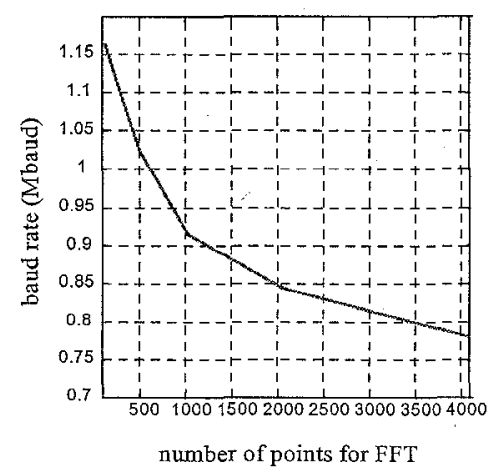

Figure 3 achievable baud rate vs. FFT length with Ariel MM96 DSP

(2) Non real time implementation of the whole system:

Rapid Prototyping can offer some advantages even if the prototype can not run in real time. In some cases, it can still be possible to optimize parameters, evaluate subjective quality and verify the possibility of implementation.

In the following, we will discuss the viability of both solutions for the construction of a Digital Television Terrestrial Broadcasting test prototype.

\section{IMPLICATIONS OF THE REDUCTION OF THE NUMBER OF SUB-CARRIERS IN AN OFDM SYSTEM}

In order to examine the effects of the reduction of the number of sub-carriers in an OFDM system, we will consider the following main points which affect the
OFDM system performance:

- channel effects

- phase noise

- synchronization

- non-linear effects

\subsection{Channel effects in OFDM}

If the transmitted signal defined in 2.2 is affected by a channel with impulse response $h(t)$, assuming that the ISI is eliminated with the time guard, the received signal is:

$$
r(n)=s(n) \otimes h(n)=\sum_{m=0}^{N-1} s(m) \cdot h(n-m)=\sum_{m=0}^{N-1} h(m) \cdot s(n-m)
$$

and it is Orthogonal Frequency Division Demultiplexed:

$$
\begin{gathered}
\frac{1}{N} \sum_{r=0}^{N-1} r(r) \cdot e^{-j \frac{2 \pi}{N} r n}=\frac{1}{N} \sum_{r=0}^{N-1} \sum_{m=0}^{N-1} h(m) \cdot s(r-m) \cdot e^{-j \frac{2 \pi}{N} r n}= \\
=\frac{1}{N} \sum_{r=0}^{N-1} \sum_{m=0}^{N-1} h(m) \sum_{l=0}^{N-1} s_{l} e^{j \frac{2 \pi}{N} l(r-m)} \cdot e^{-j \frac{2 \pi}{N} r n}= \\
=\frac{1}{N} \sum_{r=0}^{N-1} \sum_{l=0}^{N-1} s_{l} \cdot e^{j \frac{2 \pi}{N} l(r-n)} \sum_{m=0}^{N-1} h(m) \cdot e^{-j \frac{2 \pi}{N} l m}= \\
=\frac{1}{N} \sum_{l=0}^{N-1} s_{l} \cdot H(l) \sum_{r=0}^{N-1} e^{j \frac{2 \pi}{N} l(r-n)}=H(n) \cdot s_{n} \\
\text { with } H(n)=\sum_{m=0}^{N-1} h(m) \cdot e^{-j \frac{2 \pi}{N} n m}, \text { the DFT of the }
\end{gathered}
$$
channel impulse response.

This means that the channel affects the demultiplexed signal by multiplying each symbol by the frequency response corresponding to this symbol sub-carrier $H(n)$.

The OFDM signal distribution can be used to insert pilot carriers for channel estimation and correction. The channel frequency response for all the information carriers can be obtained by interpolation from the values provided by these pilots.



Figure 4 channel estimation at the receiver

The channel correction can thus be accomplished by division of the OFDD signal by an estimation of $H(n)$ (Figure 4). In order to obtain this estimation (FFT of the channel impulse response), two types of pilots are inserted in the OFDM frame: scattered and continual pilots $[5,26]$.

Scattered pilots occupy different carrier positions on each OFDM symbol, with a periodicity of 4 symbols. Continual pilots are inserted in identical fixed positions for every OFDM symbol. 
From the scattered pilot distribution, which gives the channel FFT coefficients for discrete carrier indexes and time instants, time interpolation is applied so as to obtain the coefficients for every time instant and discrete carrier indexes. This means that if in symbol $n$ a given carrier $k$ is a pilot, coefficients are obtained corresponding to carrier $k$ for all symbols.

Besides, continual pilots are used to estimate a common phase error, which can appear due to the local oscillator's phase noise and does not depend on the carrier index, but varies randomly between OFDM symbols.

The OFDM system performance has been analyzed considering the dependence of the channel effects and the pilot-based correction capabilities with the number of sub-carriers. Two channel models have been considered, which correspond to fixed reception (F1) and portable reception (P1) in the European Digital Television for Terrestrial Broadcasting system (dTTb) $[5,26]$.

\subsubsection{Inter-Symbol Interference (ISI):}

In order to cope with multipath delay spread, a Time Guard is added to the OFDM signal so as to absorb the inter-symbol interference (ISI). This Time Guard is a cyclic extension of the signal built by adding the last $N_{g}$ samples of the OFDM symbol at the beginning, giving a total OFDM symbol duration of $N+N_{g}$ samples $\left(T+T_{g}\right.$ seconds).

If the Time Guard is longer than the channel impulse response, the ISI is contained in the first $N_{g}$ samples of the OFDM symbol, which are discarded before the demultiplexion via FFT in the receiver.

However, if the Time Guard is not long enough, some ISI will be present.

This Time Guard duration is limited by the information rate: the longer the Time Guard, the lower the ratio information bits

total OFDM duration

Since the OFDM duration depends on the number of sub-carriers $N$, if the sub-carrier spacing is small (many sub-carriers), the Time Guard can be larger without affecting the information rate seriously.

The Time Guards proposed in $\mathrm{dTTb}$ for $2 \mathrm{~K}$ and $8 \mathrm{~K}$ subcarriers, defined as the ratio between the guard duration and the OFDM symbol time, are shown in Table 1. Besides, 128 sub-carriers have been considered.

Table 1 OFDM Time Guards

\begin{tabular}{||c|c|c|c|c||}
\hline & $1 / 4$ & $1 / 8$ & $1 / 16$ & $1 / 32$ \\
\hline $8 \mathrm{~K}(\mathrm{~T}=896 \mu \mathrm{s})$ & $224 \mu \mathrm{s}$ & $112 \mu \mathrm{s}$ & $56 \mu \mathrm{s}$ & $28 \mu \mathrm{s}$ \\
\hline $2 \mathrm{~K}(\mathrm{~T}=224 \mu \mathrm{s})$ & $56 \mu \mathrm{s}$ & $28 \mu \mathrm{s}$ & $14 \mu \mathrm{s}$ & $7 \mu \mathrm{s}$ \\
\hline $128(\mathrm{~T}=14 \mu \mathrm{s})$ & $3.5 \mu \mathrm{s}$ & $1.75 \mu \mathrm{s}$ & $0.875 \mu \mathrm{s}$ & $0.4375 \mu \mathrm{s}$ \\
\hline
\end{tabular}

No ISI will appear in any case when using $2 \mathrm{~K}$ or $8 \mathrm{~K}$ sub-carriers (the impulse response duration of both $F 1$ and P1 channels is $5.42 \mu \mathrm{s}$ ), but we would need to increase the Guard Time ratio up to $1 / 2(7 \mu \mathrm{s})$ to avoid ISI when using 128 sub-carriers. However this would mean a $33 \%$ decrease of the information rate, which we normally can not afford.

\subsubsection{Channel distortion without ISI}

If the ISI is eliminated with the Time Guard, the only channel distortion caused to the signal is the multiplication by the channel frequency response. This means that when the channel presents an attenuation in some frequency, the corresponding symbol will be corrupted (unless some channel correction is applied).

This effect is independent of the sub-carrier spacing, as Figure 5 shows.

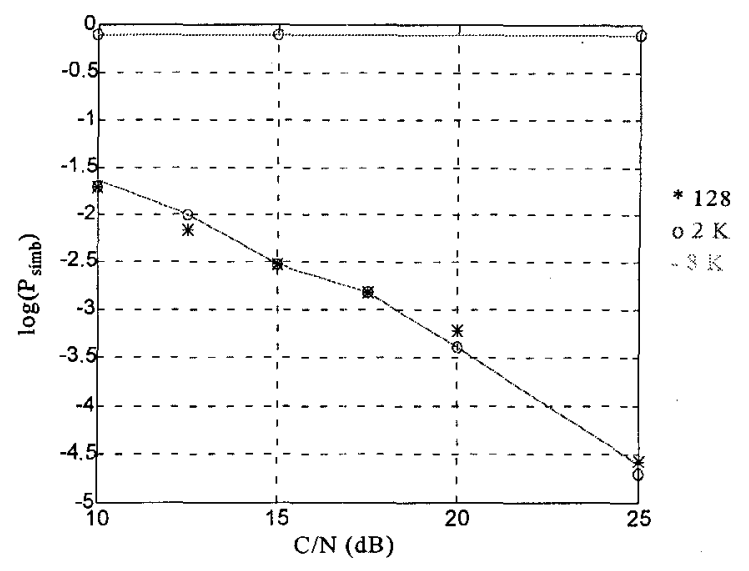

Figure 5 channel effects for $8 K, 2 K$ and 128 sub-carriers ( $P 1$ : upper curves, F1: lower curves)

\subsubsection{Channel correction capabilities}

The channel estimation is accomplished by means of interpolation using the pilot structure introduced in the OFDM frame (as explained at the beginning of this section).

The accuracy of the estimation depends strongly on the number of sub-carriers. The reason is that if this number is small, not enough pilots will be included in the frame to interpolate the channel frequency response, as it happens with the 128 sub-carrier system. Obviously the number of pilots has to be limited in order to maintain an adequate information rate.

Table 2 shows the power of the estimation error, computed this error as the difference between the channel frequency response and the channel estimation.

Table 2 power of estimation error

\begin{tabular}{||c|c|c|c|}
\hline & 128 & $2 \mathrm{~K}$ & $\mathbf{8 K}$ \\
\hline F1 channel & 0.14 & 0.03 & 0.002 \\
\hline P1 channel & 1.5 & 0.2 & 0.017 \\
\hline
\end{tabular}

Although the error is bigger when using $2 \mathrm{~K}$ than with $8 \mathrm{~K}$ sub-carriers, because less pilots can be used for 
estimation (the difference is one order of magnitude), channel correction is still possible. However, this is not the case when using only 128 sub-carriers, as shown in Figure 6.

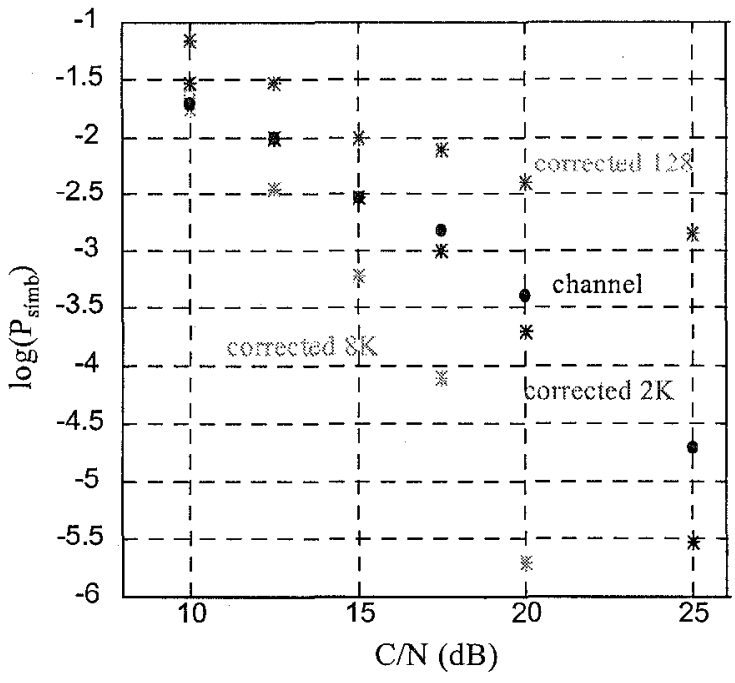

Figure 6 SER versus $\mathrm{C} / \mathrm{N}$ with and without channel correction

In this figure, Symbol Error Rate (SER) is plotted versus carrier to noise power ratio $(\mathrm{C} / \mathrm{N})$ for the $\mathrm{F} 1$ channel. The channel output SER and the corrected SER are compared.

All systems show a similar SER when no correction is applied (with ' $\mathrm{O}$ ' in Figure 6) as mentioned above (section 3.1.1), but they exhibit different correction capabilities ('*⿻丷木' in Figure 6). While the improvement is more than $5 \mathrm{~dB}$ with $8 \mathrm{~K}$, with $2 \mathrm{~K}$ is only about $2 \mathrm{~dB}$ and with 128 sub-carriers, the SER is even worse when channel correction is applied than without correction.

This means that a number of 128 sub-carriers can not be used in a multipath environment.

\subsection{Phase noise effects in OFDM}

All superheterodyne receivers use one or more local oscillators to convert an input frequency to an intermediate frequency before the signal is demodulated. Both the mixer used for converting the signal's frequency and the local oscillator will distort the signal and limit the receiver's ability to recover the modulation on a signal [27].

Although the mixer degradation can be minimized by proper design in the rest of the receiver, the phase noise introduced by the local oscillator cannot be decreased except by improving the performance of the oscillator. Therefore the importance of determining how much phase noise a receiver can admit maintaining the required performance.

Assuming that the channel is flat, the transmitted signal defined in 2.2 is only affected by phase noise $\phi(n)$ at the receiver:

$$
r(n)=s(n) \cdot e^{j \phi(n)}
$$

and it is Orthogonal Frequency Division Demultiplexed by means of a Discrete Fourier Transform:

$$
\begin{aligned}
& y(k)=\frac{1}{N} \sum_{m=0}^{N-1} r(m) \cdot e^{-j \frac{2 \pi}{N} k m}=\frac{1}{N} \sum_{m=0}^{N-1} e^{j \phi(m)} \sum_{r=0}^{N-1} s_{r} \cdot e^{j \frac{2 \pi}{N} r m} \cdot e^{-j \frac{2 \pi}{N} k m}= \\
& =\frac{1}{N} \sum_{m=0}^{N-1} e^{j \phi(m)} \sum_{r=0}^{N-1} s_{r} \cdot e^{j \frac{2 \pi}{N}(r-k) m}=\frac{1}{N} \sum_{r=0}^{N-1} s_{r} \sum_{m=0}^{N-1} e^{j \phi(m)} \cdot e^{j \frac{2 \pi}{N}(r-k) m}
\end{aligned}
$$

In order to separate the signal and noise terms, let us suppose that $\phi(m)$ is small, so that $e^{j \phi(m)} \approx 1+j \phi(m)$. In this case:

$$
\begin{aligned}
y(k) & \approx \frac{1}{N} \sum_{r=0}^{N-1} s_{r} \sum_{m=0}^{N-1} e^{j \frac{2 \pi}{N}(r-k) m}+\frac{j}{N} \sum_{r=0}^{N-1} s_{r} \sum_{m=0}^{N-1} \phi(m) e^{j \frac{2 \pi}{N}(r-k) m}= \\
& =s_{k}+\frac{j}{N} \sum_{r=0}^{N-1} s_{r} \sum_{m=0}^{N-1} \phi(m) e^{j \frac{2 \pi}{N}(r-k) m}=s_{k}+e_{k}
\end{aligned}
$$

Thus we have an error term $e_{k}$ for each sub-carrier which results from some combination of all carriers and is added to the useful signal. Let us analyze more deeply this noise contribution:

$$
\text { a) If } \mathrm{r}=\mathrm{k} \text { : }
$$

$$
\frac{j}{N} \sum_{r=0}^{N-1} s_{r} \sum_{m=0}^{N-1} \phi(m)=j \frac{s_{k}}{N} \sum_{m=0}^{N-1} \phi(m)=j \cdot s_{k} \cdot \Phi
$$

So we have a common error added to every sub-carrier which is proportional to its value multiplied by a complex number $j \Phi$, that is a rotation of the constellation.

This angle results from an average of phase noise over all of them (which implies low frequencies of phase noise spectrum) and, since it is constant for all subcarriers, it can be corrected with the information provided by pilots.

b) If $r \neq k$ :

$$
\frac{j}{N} \sum_{\substack{r=0 \\ r \neq k}}^{N-1} s_{r} \sum_{m=0}^{N-1} \phi(m) \cdot e^{j \frac{2 \pi}{N}(r-k) m}
$$

This term corresponds to the summation of the information of the $N-1$ sub-carriers each multiplied by some complex number which comes from an average of phase noise with a spectral shift. The result is also a complex number which is added to each sub-carrier's useful signal and has the appearance of white noise. It is normally known as inter-carrier interference (ICI) or loss of orthogonallity.

\subsubsection{Error performance}

Simulations have been run so as to study the influence of phase noise in the reception of OFDM signals and the phase noise error correcting capabilities of pilot-based channel correction. The assumptions and simplifications made in the previous analysis to understand the system behavior have not been applied to simulations, so results can be obtained for any phase noise and channel situations.

We have modeled phase noise as a phase modulation of the carrier. The modulating signal is a white random 
process with a uniform or gaussian pdf, which has been filtered so as to analyze the influence of the phase noise correlation characteristics in the signal quality. Results are presented using the reciprocal of the filtered phase noise bandwidth: $T_{\phi}$ and comparing it to the OFDM symbol duration: $T_{\text {OFDM }}$.

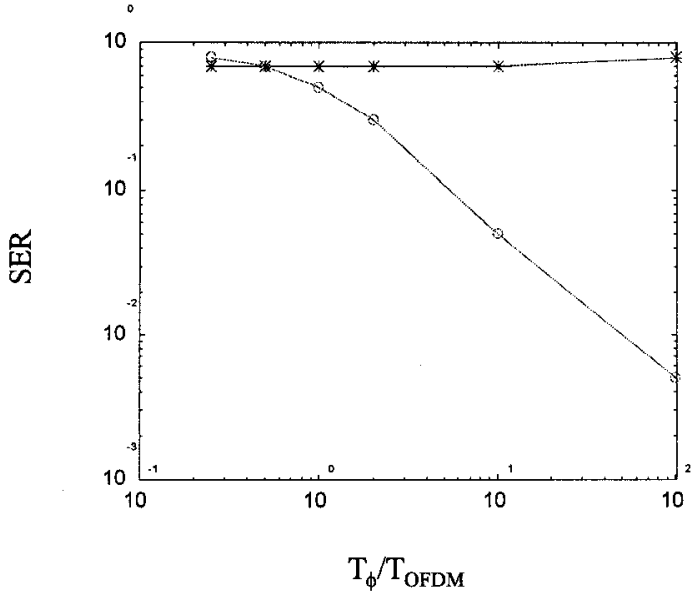

Figure 7 phase noise in OFDM: * corrupted signal, o corrected signal

Figure 7 shows the behavior of an OFDM system corrupted by uniform phase noise when using pilotbased channel correction. As it can be observed, phase noise effects can be corrected when the phase noise variations are much slower than the OFDM period. In this case the common phase error dominates over the ICI.

The same curves are obtained when considering any number of sub-carriers if the phase noise period is normalized with respect to OFDM symbol duration.

When $T_{\phi}<T_{O F D M}$, pilot based channel correction is not able to correct phase noise errors and it can even increase the SER.

If the period of phase noise variations is expressed in seconds, the results are those of Figure 8 .

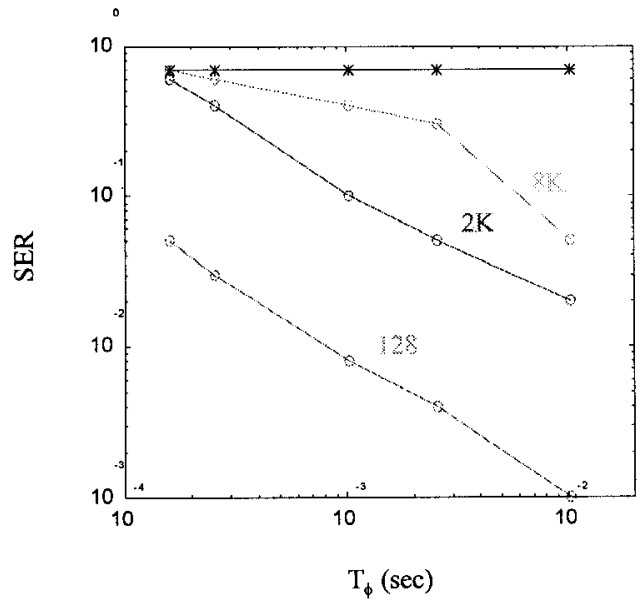

Figure 8 OFDM with different numbers of sub-carriers: * corrupted, o corrected

The symbol error rate (SER) degradation caused by phase noise is the same for $128,2 \mathrm{~K}$ and $8 \mathrm{~K}$ systems. However, the correction capabilities, as indicated above, depend on the phase noise to OFDM time ratios. It is clear that for a given phase. noise bandwidth, the performance of a 128 sub-carrier OFDM system is much better than that corresponding to a bigger number of sub-carriers, provided that the phase noise variations are much slower than the OFDM period. This means that the phase noise bandwidth should be less than $1 / 14 \mu \mathrm{s} \approx 71 \mathrm{KHz}$. Otherwise, the pilot structure will not be usable to correct phase noise errors.

\subsection{OFDM synchronization}

The OFDM synchronization system in the receiver must execute the following functions:

- Decide which number of sub-carriers is using the system $(2 \mathrm{~K} / 8 \mathrm{~K})$.

- Estimate which is the Time Guard value among the various possibilities.

\section{- Adjust the FFT starting position.}

They are accomplished taking profit of the OFDM signal correlation properties after insertion of the Time Guard.

In fact, there are $N_{g}$ samples of the signal which are repeated within an interval of $N+N_{g}$ samples. This property can be used to synchronize the receiver by making the following correlation:

$$
\operatorname{corr}(n)=\sum_{k=n-N_{I}+1}^{n} s(k) \cdot s^{*}(k-N)
$$

Where $N$ is the number of points in the transmitter IFFT (number of sub-carriers) and $N_{I}$ is a parameter which must be chosen theoretically equal to and never bigger than the Guard Time $N_{g}$.

In the absence of noise and multipath, this correlation presents the aspect shown in Figure 9. When $N_{I}=N_{g}$, there is a peak at the beginning of the useful OFDM symbol. The peak is replaced by a flat top of duration $N_{I}+N_{g}$ when $N_{I}<N_{g}$, which ends also at the beginning of the useful symbol.

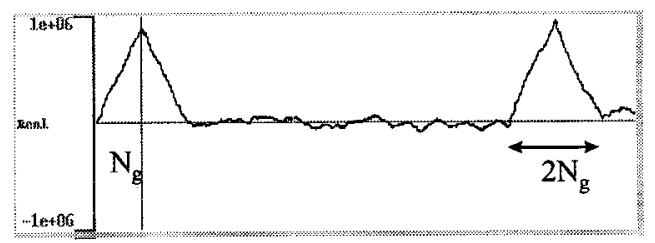

(a) $N_{\Gamma}=N_{g}=256$

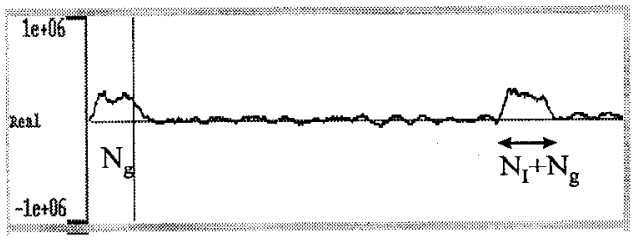

(b) $N_{i}=64, N_{g}=256$

Figure 9 OFDM (2K) correlation with different $N_{t}$

Noise enhances the correlation levels between the peaks 
(Figure 10). Multipath has more noticeable effects as Figure 11 shows for an OFDM signal after P1 channel. The peak is broadened due to the channel delay spread.

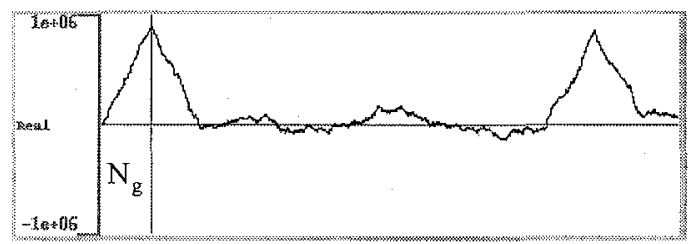

Figure $102 \mathrm{~K}$-OFDM: $N_{l}=N_{g}=256, \mathrm{C} / \mathrm{N}=3 \mathrm{~dB}$

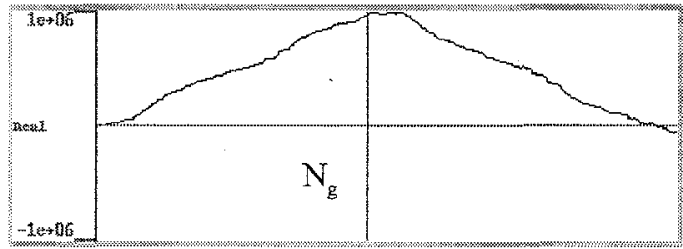

Figure $112 \mathrm{~K}$-OFDM: $N_{l}=N_{g}=256$, after P1 channel (detail of the peak)

If the number of sub-carriers $N$ used in the correlation is different from the actual one in the transmitter, no peaks will appear (Figure 12).

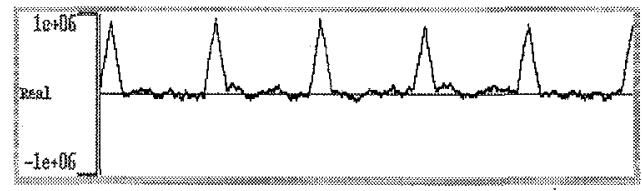

(a) correlation with $N=2 \mathrm{~K}$

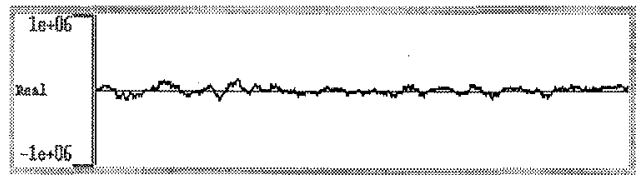

(b) correlation with $N=8 \mathrm{~K}$



(c) correlation with $N=1 \mathrm{~K}$

Figure 12 correlation of 2K-OFDM with different values of $N$

In order to decide how many sub-carriers are being used, two correlations are calculated during several symbols with the two possible $N$ values and $N_{I}$ equal to the minimum $N_{g}$ expected. The maximum values are compared and if they exceed a certain threshold, the $\mathrm{N}$ value which produced the highest maximum is chosen.

The Guard Time is estimated by finding four consecutive peaks in the correlation calculated with the just estimated $N$ value and $N_{I}$ equal to the minimum $N_{g}$ expected for this $N$ value. The distance between the first and the fourth peak indicates the $N_{\mathrm{g}}$ value:

$$
N_{g}=\left(\max _{4}-\max _{1}-3 N\right) / 3
$$

Once that $N$ and $N_{g}$ are known, a last correlation is calculated with these parameters, and the FFT starting point is given by the peak position. A finer adjust can be achieved later by controlling the A/D converter clock.

The precise synchronization in the receiver depends strongly on the alteration of the correlation properties caused by the presence of noise and multipath. If the peaks can not be clearly identified or their position changes, the synchronization will fail.

Figure 13 shows that the correlation in an OFDM system with 128 sub-carriers is much more sensible to AWGN than a $2 \mathrm{~K}$ one. Since the difference between the peaks and the correlation floor is much lower in the first one, the noise level can hide the peaks.



(a) 128-OFDM with $\mathrm{C} / \mathrm{N}=3 \mathrm{~dB}$

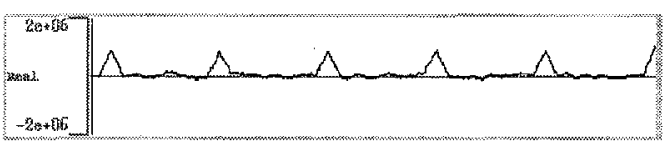

(b) 2K-OFDM with $\mathrm{C} / \mathrm{N}=3 \mathrm{~dB}$

Figure 13 correlation sensitivity to AWGN

The sensitivity to multipath delay spread is also higher in a 128 sub-carrier system. In Figure $14,2 \mathrm{~K}$ and 128 are compared when using the same $N_{g} / N$ value, showing that multiple peaks are generated in the 128 system.

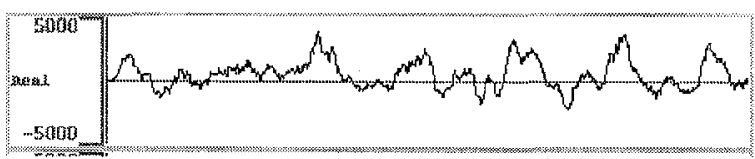

(a) 128-OFDM with $N_{g}=16$



(b) $2 \mathrm{~K}$-OFDM with $N_{g}=256$

Figure 14 correlation sensitivity to multipath

For these reasons, synchronization of a system with a low number of sub-carriers using the OFDM correlation is more a difficult task.

\subsection{Non-linear effects in OFDM}

Efficient use of power amplifiers implies a working point close to saturation, which causes a non-linear behavior and thus a degradation in the signal quality.

Since the OFDM signal is composed of many independently modulated sub-carriers, it presents a Gaussian distribution (Central Limit Theorem) with high peak to medium value ratios. This fact makes it 
very sensitive to non-linear effects.

Provided that it is big enough to satisfy this theorem $(N>100)$, the number of sub-carriers does not affect the peak to medium value ratio. In fact, simulated OFDM signals (1500 symbols) exhibit a $12.9 \mathrm{~dB}$ variation when the modulation is QPSK and $13.2 \mathrm{~dB}$ when it is $16-\mathrm{QAM}$, independently of the value of $N(128,2 \mathrm{~K}$, $8 \mathrm{~K})$.

The error performance degradation caused by a nonlinear amplifier has been studied via simulation, using complex envelope representation and the AM/AM characteristics of the amplifier. The results are presented in Figure 15 and Figure 16 for $2 \mathrm{~K}$ and $8 \mathrm{~K}$ systems.

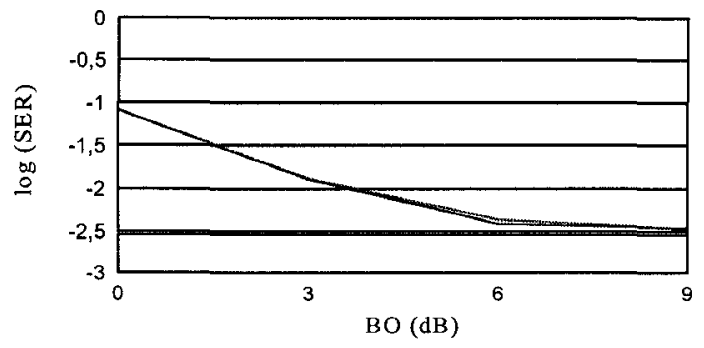

Figure 15 performance of 16-QAM-OFDM

A back off of $6 \mathrm{~dB}$ is needed to demodulate a 16-QAM signal with a degradation in $\log (\mathrm{SER})$ of only 0.13 with respect to an ideal situation for a $\mathrm{C} / \mathrm{N}$ ratio of $16 \mathrm{~dB}$.

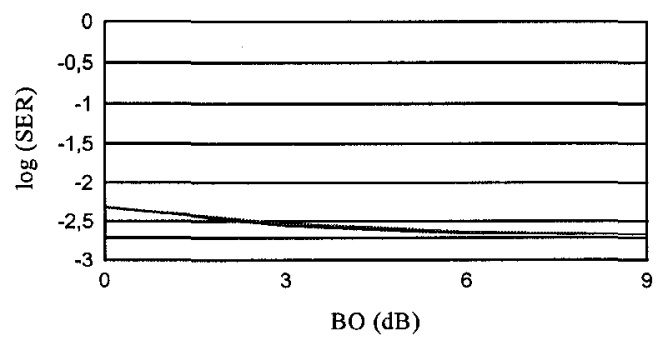

Figure 16 performance of QPSK-OFDM

When the modulation is QPSK and $\mathrm{C} / \mathrm{N}=9 \mathrm{~dB}$, a $3 \mathrm{~dB}$ back off is enough, with a degradation of 0.16 in $\log$ (SER).

In both cases, the number of sub-carriers does not affect the error performance.

\subsection{Reduction of the number of sub-carriers in an OFDM system}

To summarize, the conclusions of the preceding sections are:

a) Simulations show that large Time Guard intervals are needed to fight inter-symbol interference (ISI) caused by multipath. For this reason, in order to maintain a reasonable information rate, a system with a low number of sub-carriers should be avoided.

When ISI is conveniently suppressed, the channel frequency response has to be estimated in order to correct the amplitude and phase variations caused to the signal. If pilot-based interpolation is used, a number of sub-carriers are needed to accommodate enough pilots for the estimation. This means that again 128 subcarriers are not sufficient to provide a SER improvement.

b) Depending on the phase noise bandwidth expected in the system, two situations can be observed:

- given that phase noise variations are not very fast related to the OFDM signal, a greater number of sub-carriers will lead to a worse performance.

- if $T_{\phi}<T_{O F D M}$, the same performance is achieved regardless to the sub-carrier spacing.

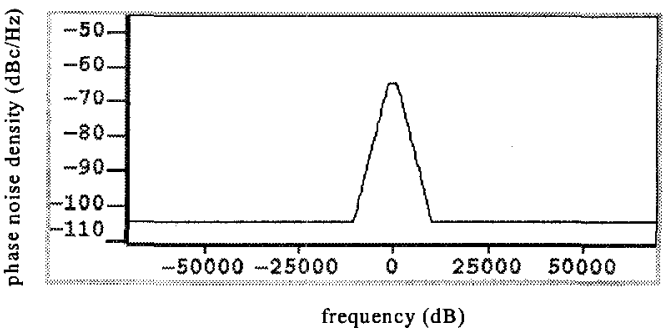

Figure $17 \mathrm{dTTb}$ phase noise mask

In situations when phase noise bandwidth is less than 71 $\mathrm{KHz}$, the error performance of a system with 128 subcarriers will be better than that of a system using $2 \mathrm{~K}$ or $8 \mathrm{~K}$. This will be normally the case in the system under study, as it can be deduced from the phase noise mask defined within the dTTb project (Figure 17).

c) Synchronization functions are harder in a $128 \mathrm{sub}$-carrier system, because the correlation used for this purpose is much more sensitive to AWGN and multipath.

d) OFDM signals constrain the power amplifiers back off to be higher than in single carrier applications. However, the non-linear effects do not depend on the number of sub-carriers used, given that the number is greater than 100 .

These points imply that a prototype with 128 subcarriers will not provide the same information as a $2 \mathrm{~K}$ or $8 \mathrm{~K}$ one about the main impairments which affect the OFDM signal and as a consequence, the first solution suggested to the real-time Rapid Prototyping of this system would not be acceptable.

\section{NON REAL TIME IMPLEMENTATION OF THE WHOLE SYSTEM}

The following process has been carried out to obtain a prototype of the whole system:

(1) Simulation models for the basic elements in transmitter and receiver

- Inner coding: convolutional codes with different coding rates obtained by puncturing of the mother $1 / 2$ code $[8,9]$. They are decoded using the Viterbi algorithm with the same decoder to every punctured code and a previous insertion of dummy bits.

- Inner interleaving specified in $\mathrm{dTTb}$. 
- Digital modulation: multi-resolution [7] 4,8 or 16-QAM combined with inner coding.

- OFDM-OFDD [22] with $2 \mathrm{~K}$ and $8 \mathrm{~K}$ subcarriers using frequency guards as a protection against adjacent channel interference (ACI) and time guards to fight inter-symbol interference (ISI).

(2) Simulation model for the channels. Channel estimation and synchronization

Two channel models have been implemented for simulation, which correspond to those suggested within the $\mathrm{dTTb}$ project to evaluate the system performance: $\mathrm{F} 1$ for fixed reception and $\mathrm{P} 1$ for portable reception $[5,26]$.

Pilot sub-carriers have been introduced in the OFDM frame and channel correction by interpolation of the channel response (section 3.1) has been studied and optimized $[5,26]$.

Simulation models for synchronization of the receiver using the OFDM correlation properties have been created.

\section{(3) DSP implementation of the basic elements in transmitter and receiver}

After optimization of the above described transmitter and receiver basic functions, $\mathrm{C}$ code has been generated to be compiled and executed in an Ariel MM-96 board [23] containing two Motorola DSP96002 [24].

Simulated and DSP signals have been compared to check the correctness of the prototype and verify the achievement of the system specifications. Modulation and coding parameters have been optimized in a feedback process between simulation and DSP prototype.

(4) DSP implementation of the channel estimation and synchronization subsystems

In the same way $\mathrm{C}$ code has been generated and verified for the channel estimation (and correction) and synchronization subsystems.

The transmitter runs in an MM-96 Ariel board [23]. It reads MPEG2 data from a file (or any general data type), processes the base band signal (coding, digital modulation and OFDM) and writes the signal back to a file.

The receiver, in another MM-96, reads this file (which can be modified in the simulator to include signal distortions such as multipath or phase noise), realizes the synchronization process and then obtains the output data after channel correction, demodulation and decoding.

In this case, Rapid Prototyping can offer some advantages even though the prototype can not run in real time. It is possible to optimize parameters and verify the possibility of implementation. Besides, subjective qualities can be observed, since MPEG2 images obtained in the receiver after channel impairments can be compared to the transmitted ones.

\section{CONCLUSIONS}

There is an increasing interest in Rapid Prototyping of communications systems, with the main objective of reducing the design cycle and improving the products quality. However, Rapid Prototyping of a complex system presents some difficulties, which depend on the system characteristics.

We have applied this concepts to the construction of a prototype for the European Digital Television for Terrestrial Broadcasting System (dTTb) whose transmission technique (Orthogonal Frequency Division Multiplexing) has been introduced. After identifying the problems associated with the construction of a prototype, two different solutions have been proposed and discussed:

- Scaling the number of sub-carriers in the OFDM system so that a real time implementation is possible.

- Non real time implementation of the whole system.

The first solution has been discarded, since a scaled system would not provide the same information about the main signal impairments as the real one.

The second solution has been adopted, and a prototype has been presented which allows us to optimize parameters, verify the possibility of implementation and observe subjective qualities.

\section{REFERENCES}

[1] 'Grand Alliance HDTV System Specification', version 2.0, December 1994

[2] Y. Wu, B. Caron: 'Digital Televison Terrestrial Broadcasting', IEEE Communications Magazine, vol. 32, no. 5: 46-52, May 1994

[3] EBU V4/MOD-B: 'Baseline specification for the European system for broadcasting digital television, sound and data services by satellite in the $11 / 12 \mathrm{GHz}$ bands' Technical Report, European Broadcasting Union, Geneva, Switzerland, November 1993

[4] EBU/CENELEC/ETSI JTC: 'Digital broadcasting systems for television, sound and data services, framing structure, channel coding and modulation for digital terrestrial television', January 1996, TM 1545 rev. 2

[5] dTTb Module 3: 'System Specification for the Second dTTb Demonstrator', Version 2.2, February 1996

[6] ISO/IEC 13818 Part 1, 2, 3: 'Coding of moving Pictures and associated audio', November 1994

[7] H. Imai, S. Hirakawa, 'A new multilevel coding method using error correcting codes', IEEE Transactions on Information Theory, IT-23(3): 371-377, 1977

[8] J. Hagenauer: 'Rate-compatible puctured convolutional codes (RCPC codes) and their applications', IEEE Transactions on Communications, 36(4): 389-400, April 1988

[9] Y. Yasuda, K. Kashiki, Y. Hirata: 'High-Rate Punctured Convolutional Codes for Soft Decision Viterbi Decoding', IEEE Transactions on Communications, vol. COM-32, no. 3: 315-317, March 1984 
[10] J. Mitola: 'The Software Radio Architecture', IEEE Communications Magazine, vol. 33, no. 5: 26-38, May 1995

[11] R. H. Hosking: 'Digital Receivers Bring DSP to Radio Frequencies', Proc. ICSPAT'95: 465-468, Boston, October 1995

[12] J. A. Wepman: 'Analog-to-Digital Converters and their Applications in Radio Receivers', IEEE Communications Magazine, vol. 33, no. 5: 39-45, May 1995

[13] CECASE and TISL Laboratories, Univ. of Kansas: 'Digital Signal Processing (DSP) Rapid Prototyping for Military Communtication Systems', Final Technical Report, Contract \# F30602-93-C-0109, November 1994.

[14] G. Gupta, S. Srinivas, C. Neophytou: 'Design and Implementation of a Digital Spread Spectrum Wireless Modem using Rapid Prototyping Concepts', Proc. $5^{\text {th }}$ International Conference on Signal Processing Applications and Technology ICSPAT'94: 18-21, Dallas, October 1994

[15] H. Chakravarthula, S. Srinivas, G. Prescott, T. Johnson, S. Tyler: 'Parallel implementation of the CELP speech compression algorithm on a network of TMS320C40 processors', Proc. $6^{\text {th }}$ International Conference on Signal Processing Applications and Technology ICSPAT'95: 1888-1891, Boston, October 1995.

[16] C. P. Neophytou: 'Design and Implementation of a Digital Modem using Minimum Shift Keying on the TMS320C30 and 'C40 Digital Signal Processors', M. S. Thesis, Dept. of Electrical Engineering and Computer Science, University of Kansas, 1994.

[17] H. N. Chakravarthula: 'Implementation of a real-time 4800 bps CELP algorithm on the TMS320C40 processor', M. S. Thesis, Dept. of Electrical Engineering and Computer Science, University of Kansas, 1993.

[18] Isikli, Engels, Lauwereins, Peperstraete: 'Rapid
Prototyping of an Adaptive Beamformer for GSM Base Stations', Proc. of the IASTED International Conference on Signal and Image Processing and Applications, Annecy, France, June 1996.

[19] Leonard J. Cimini, 'Anallysis and Simulation of a Digital Mobile Channel Using Orthogonal Frequency Division Multiplexing', IEEE Trans. on Communications, Vol. COM-33, No. 7, July 1985.

[20] B. Le Floch, R. H. Lassalle, D. Castelain, 'Digital Sound Broadcasting to Mobile Receivers', IEEE Trans. on Consumer Electronics, Vol. 35, No. 3, August 1989.

[21] K. Jacobsen, J. Cioffi, 'An Efficient Digital Modulation Scheme for Multimedia Transmission on the Cable Television Network', 43 rd $^{\text {rd }}$ Annual National Cable Television Association (NTCA) Convention and Exposition, New Orleans, LA, May 1994.

[22] S. B. Weinstein, P. M. Ebert, 'Data Transmission by Frequency-Division Multiplexing Using the Discrete Fourier Transform', IEEE Trans. on Communication Technology, Vol. COM-19. No. 5. October 1971.

[23] 'MM-96/DSP-96 Release Notes', release 1.21, Ariel Corporation, July 1993

[24] 'DSP96002 IEEE Floating-Point Dual-Port Processor: User's Manual', Motorola, 1989

[25] T. L. Freeman, C. Phillips: 'Parallel Numerical Algorithms'.

[26] A. G. Armada, M. Calvo, L. de Haro,: 'Pilot-Based Channel correction in an OFDM Communication System', Proc. of Commsphere'97, Lausanne (Switzerland) 1997.

[27] Rhode, U. 'Digital PLL frequency synthesizers. Theory and Design', Ed. Prentice Hall, 1983.



Ana García Armada was born in Santiago de Compostela, Spain, in 1970.

She received the Telecommunication Engineer degree from the ETSIT, Technical University of Madrid in July 1994.

She has been involved in Simulation and Rapid Prototyping of the European Digital TV Terrestrial Broadcasting System (dTTb) based on COFDM signals as a Ph.D. student.

Miguel Calvo Ramón was born in Pueyo de Jaca, Huesca, Spain in 1949.

He got the Telecommunication Engineer degree from the ETSIT of the Technical University of Madrid in 1974 and the Ph.D. degree from the same University in 1979. He works as Professor in the Signals, Systems and Radiocommunications department.

Has co-authored a number of papers in technical reviews and contributed in a number of international conferences. He wrote a chapter in the book "Reflector and Lens Antennas. Analysis and Design using Personal Computers", C.J. Sletten editor, Artech House 1988. 